International Journal of Advancement in Life Sciences Research
Online ISSN: $2581-4877$
journal homepage http://ijalsr.org

Original Article

\title{
Status of Ichthyofaunal Diversity of River Ganga in Malda District of West Bengal, India
}

\author{
Suchismita Medda ${ }^{1}$, Santi Ranjan Dey ${ }^{2^{\star}}$ \\ ${ }^{1}$ Mohiary Ranibala Kundu Choudhury Balika Vidyalaya, Andul-Mouri, Howrah, West Bengal 711302, India. \\ ${ }^{2}$ Department of Zoology, Rammohan College, Kolkata, West Bengal 700009, India. \\ ${ }^{*}$ Correspondence E-mail : srdey1@rediffmail.com
}

\begin{abstract}
The river Ganges is the largest river in India and the fifth longest in the world. Although, many studies on fish ecology and systematic have been conducted largely to improve fisheries but fish diversity and their distribution pattern from conservation point of view have never been adequately addressed in the Ganges River. The objective of present study was to explore the present Ichthyofaunal diversity of the stretch of Ganga at Malda district of West Bengal. The result showed that 69 freshwater fish species belonging to 9 Orders, 24 Families was found in Ganga stretch of Malda District of West Bengal, India.
\end{abstract}

Keywords: Malda; Ganga; Ichthyofauna; IUCN.

\section{Introduction}

Aquatic ecosystem is divided into lotic and lentic system based on flow. Lotic system is mainly comprised of river and sea and lentic system includes Beel, Lake, pond etc. Except sea all these water bodies contain freshwater ecosystem. Riverine Ecosystem is one of the most important harbours of freshwater fish species on earth. Fish is very important among vertebrates with respect to its socioeconomic and nutritional value as it provides protein, minerals and livelihood for many people (Jaya et al, 2020). The river Ganges is the largest river in India and the fifth longest in the world. Although, many studies on fish ecology and systematic have been conducted largely to improve fisheries but fish diversity and their distribution pattern from conservation point of view have never been adequately addressed in the Ganges River (Sarkar et al, 2012). Hamilton (1822) described 272 fish species throughout the stretches of Ganga from source of origin at Himalaya to the estuary at Bay of Bengal (Singh and Johal 2009). Most of the studies on River Ganga have been conducted on upper and middle stretches of Ganga (Kumar et al, 2019). The Ganga river harbors richest fish diversity in Indian subcontinent (Bilgrami et al, 1992; Dwivedi et al, 2016). The Ganga river fishery resources also provide a wide range of other ecosystem services (Pathak et al, 2014; Dwivedi et al, 2016). The Ganga river also supports small aquatic ecosystem for their flora and fauna diversity. Some part of the lower Ganga flows through the West Bengal before merging into Bay of Bengal. Due to presence of Farakka Barrage on the river Ganga in Malda district, the river appears to be huge but near stagnant in nature. The barrage also create a barrier in the natural mixing and migration of fish. The objective of present study is to explore the present Ichthyofaunal diversity of the stretch of Ganga at Malda district of West Bengal which will create a baseline data for comparison of Ichthyofauna in future. 


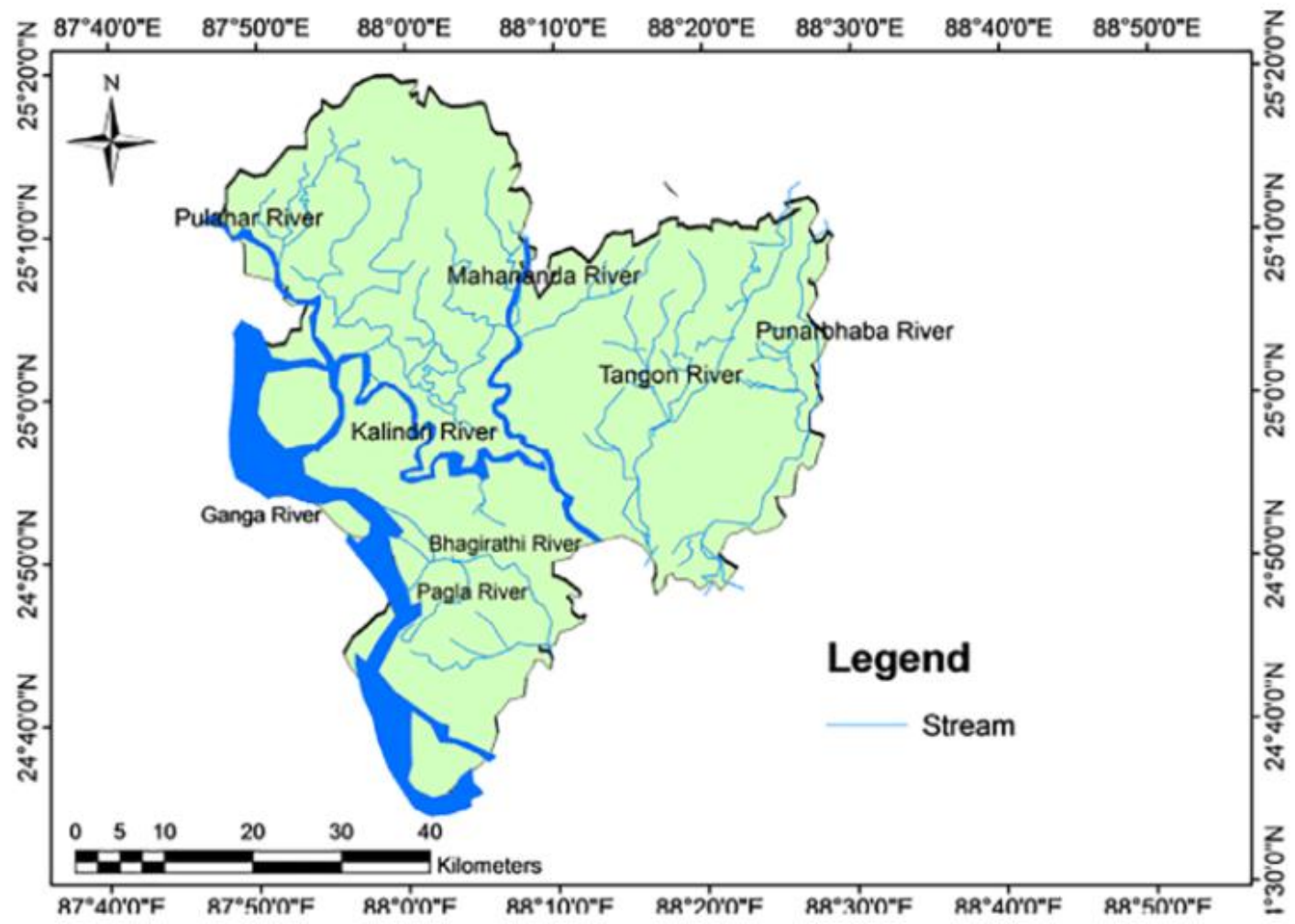

\section{Materials and Methods}

Fig. 1. Stretch of River Ganga, District Malda, West Bengal

The river Ganga was surveyed from $24^{\circ} 52^{\prime} 15^{\prime \prime} N$ N 87 $58^{\prime} 17^{\prime \prime}$ E to $24^{\circ} 51^{\prime} 36^{\prime \prime} N$ $87^{\circ} 58^{\prime} 17^{\prime \prime} \mathrm{E}$ in the Pre-monsoon, Monsoon and Post Monsoon periods for 5 years (20142019). The local markets were also surveyed for the information about fish. The fishermen associated with the river were contacted, interviewed with specific questions and their catch were analysed for collection of fishes. The collected fish were identified, photographed and preserved. Taxonomic Identification was done primarily from the books of Day, F (1876), Jayaram, K.C. (1981), Talwar and Jhingran (1991) and Barman, R. (2007). The fish fauna has been arranged taxonomically according to the classification of Jayaram, K.C. (1981). Status of the species was also studied from the data of global (IUCN) abundance status from the conservation point of view.

\section{Results}

Table 1: Ichthyofauna of Ganga River in Malda District with local name and IUCN status

\begin{tabular}{|l|l|l|}
\hline Name of Fishes & Local name & IUCN status (Global) \\
\hline $\begin{array}{l}\text { Order: Clupeiformes } \\
\text { Family: Clupeidae }\end{array}$ & Khoira & $\begin{array}{l}\text { Least Concern (LC) (Decreasing) } \\
\text { Date assessed: 06 October 2009 }\end{array}$ \\
\hline $\begin{array}{l}\text { Gudusia chapra(Hamilton, 1822) } \\
\text { Gonialosa manmina (Hamilton, 1822) }\end{array}$ & Chapila & $\begin{array}{l}\text { Least Concern (LC) } \\
\text { Date assessed: 06 October 2009 }\end{array}$ \\
\hline Family: Engraulidae & & $\begin{array}{l}\text { Least Concern (LC) } \\
\text { Date assessed: 04 December 2019 }\end{array}$ \\
\hline Setipinna phasa (Hamilton, 1822) & Fasa & \\
\hline $\begin{array}{l}\text { Order: Osteoglossiformes } \\
\text { Family: Notopteridae }\end{array}$ & & Least Concern (LC) \\
\hline Notopterus notopterus (Pallas, 1769) & Foli & \\
\hline
\end{tabular}




\begin{tabular}{|c|c|c|}
\hline & & $\begin{array}{l}\text { (Stable) Date assessed: } 30 \text { August } \\
2019\end{array}$ \\
\hline Chitala chitala (Hamilton, 1822) & Chital & $\begin{array}{l}\text { Near Threatened (NT); } \\
\text { assessed: } 28 \text { May } 2010\end{array}$ \\
\hline \multicolumn{3}{|l|}{ Order: Cypriniformes } \\
\hline \multicolumn{3}{|l|}{ Family: Cyprinidae } \\
\hline Salmostoma bacaila (Hamilton, 1822) & Chela & $\begin{array}{l}\text { Least } \quad \text { Concern }(\mathrm{LC}) \\
\text { assessed: } 17 \text { March } 2011\end{array}$ \\
\hline $\begin{array}{l}\text { Hypophthalmichthys } \\
\text { (Valenciennes, 1844) }\end{array}$ & Silver carp & $\begin{array}{l}\text { Near Threatened }(\mathrm{NT}) \text {; } \\
\text { assessed: } 20 \text { January } 2011\end{array}$ \\
\hline Cabdio morar (Hamilton, 1822) & Morari/Piuli & $\begin{array}{l}\text { Least Concern } \quad(\mathrm{LC}) \\
\text { assessed: } 09 \text { October } 2009\end{array}$ \\
\hline $\begin{array}{l}\text { Amblypharyngodon mola } \\
\text { 1822) }\end{array}$ & Mourala & 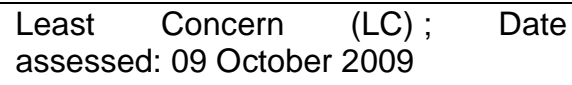 \\
\hline Barilius barila (Hamilton, 1822) & Korsa/ Tila & $\begin{array}{l}\text { Least Concern } \quad(\mathrm{LC}) ; \\
\text { assessed: } 22 \text { January } 2010\end{array}$ \\
\hline Cyprinus carpio(Linnaeus, 1758) & $\begin{array}{l}\text { American Rui, } \\
\text { Mirror carp }\end{array}$ & $\begin{array}{l}\text { Vulnerable (VU); Date assessed: } 1 \\
\text { January,2008 } \\
\text { (Exotic) }\end{array}$ \\
\hline Puntius chola (Hamilton, 1822) & Punti & \multirow{2}{*}{$\begin{array}{lcc}\text { Least Concern }(\mathrm{LC}) ; & \text { Date } \\
\text { assessed: } 20 \text { March } 2010 & \\
\text { Least Concern }(\mathrm{LC}) ; & \text { Date } \\
\text { assessed: } 22 \text { March } 2010 & \\
\end{array}$} \\
\hline Puntius conchonius(Hamilton, 1822) & Punti & \\
\hline Puntius puntio(Hamilton, 1822) & Punti & Not Evaluated \\
\hline Puntius sophore(Hamilton, 1822) & Puti & $\begin{array}{lcc}\text { Least } \quad \text { Concern }(\mathrm{LC}) ; & \text { Date } \\
\text { assessed: } 20 \text { March } 2010 & \\
\end{array}$ \\
\hline Puntius terio(Hamilton 1822) & Puti & $\begin{array}{cccc}\text { Least } & \text { Concern } & (\mathrm{LC}) ; & \text { Date } \\
\text { assessed: } 18 \text { March } 2010 & \\
\end{array}$ \\
\hline Pethia ticto (Hamilton, 1822) & Titputi & $\begin{array}{lll}\text { Least Concern }(\mathrm{LC}) ; & \text { Date } \\
\text { assessed: } 22 \text { March } 2010\end{array}$ \\
\hline Osteobrama cotio cotio (Hamilton, 1822) & Bojonmuri & $\begin{array}{llll}\text { Least } & \text { Concern } & (\mathrm{LC}) ; & \text { Date } \\
\text { assessed: } 09 \text { October } 2009 & \end{array}$ \\
\hline Labeo bata (Hamilton, 1822) & Bata & $\begin{array}{lcc}\text { Least Concern }(\mathrm{LC}) ; & \text { Date } \\
\text { assessed: } 17 \text { March } 2011 & \\
\end{array}$ \\
\hline Labeo calbasu (Hamilton, 1822) & Kalbaus & 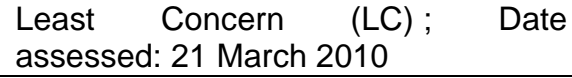 \\
\hline Labeo rohita (Hamilton, 1822) & Rui & $\begin{array}{lcc}\text { Least Concern }(\mathrm{LC}) ; & \text { Date } \\
\text { assessed: } 20 \text { March } 2010 & \\
\end{array}$ \\
\hline Cirrhinus mrigala (Hamilton, 1822) & Mrigel & $\begin{array}{lcc}\text { Least Concern }(\mathrm{LC}) ; & \text { Date } \\
\text { assessed: } 21 \text { March } 2010 & \\
\end{array}$ \\
\hline Cirrhinus reba(Hamilton, 1822) & Rai khor & $\begin{array}{lcc}\text { Least } & \text { Concern } & (\mathrm{LC}) ; \\
\text { assessed: } & 29 & \text { September } 2010\end{array}$ \\
\hline Gibelion catla (Hamilton, 1822) & Katla & 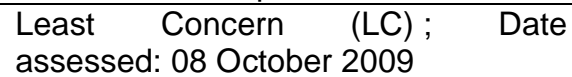 \\
\hline $\begin{array}{l}\text { Ctenopharyngodon idella(Valenciennes, } \\
1844)\end{array}$ & Grass carp & Not Evaluated (Exotic) \\
\hline Garra annandalei (Hora, 1921) & Bhola & \multirow{2}{*}{ assessed: 09 October 2009} \\
\hline Family: Cobitidae & & \\
\hline Acanthocobitis botia (Hamilton, 1822) & Balichata & 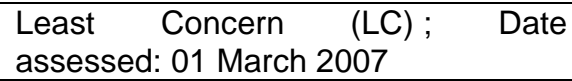 \\
\hline Botia lohachata (Chaudhuri, 1912) & Boumach & Not Evaluated (NE) \\
\hline $\begin{array}{l}\text { Lepidocephalichthys guntea (Hamilton, } \\
\text { 1822) }\end{array}$ & Gunte & \multirow[t]{2}{*}{$\begin{array}{lll}\text { Least Concern } \quad(\mathrm{LC}) ; & \text { Date } \\
\text { assessed: } 06 \text { March } 2012\end{array}$} \\
\hline Order: Siluriformes & & \\
\hline
\end{tabular}


Int J Adv Life Sci Res. Volume 4(2)14-22

\begin{tabular}{|c|c|c|}
\hline Family: Bagridae & & \\
\hline Rita rita (Hamilton, 1822) & Ritha & $\begin{array}{lcc}\text { Least } & \text { Concern } & (\mathrm{LC}) \\
\text { assessed: } & 26 \text { March } 2010\end{array}$ \\
\hline Mystus gulio (Hamilton, 1822) & Gulsatengra & $\begin{array}{l}\text { Least } \quad \text { Concern } \quad(\mathrm{LC}) \text {; } \\
\text { assessed: } 11 \text { August } 2019\end{array}$ \\
\hline Mystus vittatus (Bloch, 1794) & Sona tengra & $\begin{array}{l}\text { Least Concern } \quad(\mathrm{LC}) ; \\
\text { assessed: } 05 \text { October } 2009\end{array}$ \\
\hline Mystus tengara (Hamilton, 1822) & Bojretengra & $\begin{array}{lcc}\text { Least } & \text { Concern } & (\mathrm{LC}) \\
\text { assessed: } & \text { 05 October } 2009\end{array}$ \\
\hline Sperata aor (Hamilton, 1822) & Aar & $\begin{array}{l}\text { Least Concern (LC) ; } \\
\text { assessed: } 19 \text { March } 2011\end{array}$ \\
\hline Family: Siluridae & & \\
\hline Ompok bimaculatus (Bloch, 1794) & Deshipabda & $\begin{array}{lll}\text { Near Threatened } & \text { (NT); } & \text { Date } \\
\text { assessed: } 13 \text { October } 2009 & \\
\end{array}$ \\
\hline Wallago attu (Bloch and Schneider, 1801) & Boal & $\begin{array}{l}\text { Vulnerable (VU); Date assessed: } 12 \\
\text { August } 2019\end{array}$ \\
\hline Family: Schilbeidae & & \\
\hline Ailia coila(Hamilton, 1822) & Banspata/kajli & $\begin{array}{lccc}\text { Near } & \text { Threatened } & \text { (NT); } & \text { Date } \\
\text { assessed: } 21 \text { September } 2010 & \end{array}$ \\
\hline Pachypterus atherinoides (Bloch, 1794) & $\begin{array}{l}\text { Paloatengra/ } \\
\text { Pat tengra }\end{array}$ & $\begin{array}{lccc}\text { Least } & \text { Concern } & (\mathrm{LC}) ; & \text { Date } \\
\text { assessed: } 13 \text { October } 2009 & \\
\end{array}$ \\
\hline Clupisoma garua (Hamilton, 1822) & Ghero & $\begin{array}{lcc}\text { Least } & \text { Concern } & (\mathrm{LC}) \\
\text { assessed: } 13 \text { October } 2009\end{array}$ \\
\hline Eutropiichthy svacha (Hamilton, 1822) & Bacha & $\begin{array}{lcc}\text { Least } & \text { Concern } & (\mathrm{LC}) \\
\text { assessed: } 13 \text { October } 2009\end{array}$ \\
\hline Silonia silondia (Hamilton, 1822) & Silone & $\begin{array}{l}\text { Least Concern }(\mathrm{LC}) \text {; } \\
\text { assessed: } 01 \text { March } 2007\end{array}$ \\
\hline Family: Pangasiidae & & \\
\hline Pangasius pangasius (Hamilton, 1822) & Pangas & $\begin{array}{l}\text { Least } \quad \text { Concern } \quad(\mathrm{LC}) \\
\text { assessed: } 13 \text { October } 2009\end{array}$ \\
\hline$\frac{\text { Pangasianodon hypophthalmus (Sauvag, }}{1878)}$ & Pangas & $\begin{array}{l}\text { Endangered (EN) ; Date assessed: } 19 \\
\text { January } 2011\end{array}$ \\
\hline Family: Amblycipitidae & & \\
\hline Amblyceps apangi (Nath and Dey, 1989) & Botsingi & $\begin{array}{lccc}\text { Least } & \text { Concern } & (\mathrm{LC}) ; & \text { Date } \\
\text { assessed: } 16 \text { December } 2009 & \\
\end{array}$ \\
\hline Family: Sisoridae & & \\
\hline Bagarius bagarius(Hamilton,1822) & Bagh aar & $\begin{array}{l}\text { Near Threatened (NT); Date } \\
\text { assessed: } 13 \text { October } 2009\end{array}$ \\
\hline Conta conta(Hamilton, 1822) & Contaaar & $\begin{array}{l}\text { Data deficient }(\mathrm{DD}) ; \\
\text { assessed: } 12 \text { October } 2009\end{array}$ \\
\hline Pseudolaguvia shawi (Hora, 1921) & Tel gagor & $\begin{array}{l}\text { Least Concern }(\mathrm{LC}) ; \\
\text { assessed:12 October } 2009\end{array}$ \\
\hline Glyptothorax telchitta(Hamilton, 1822) & Telchita & 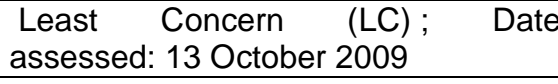 \\
\hline Family: Heteropneustidae & & \\
\hline Heteropneustes fossilis(Bloch, 1794) & Shingi & $\begin{array}{l}\text { Least Concern } \quad(\text { LC) } \\
\text { assessed: } 11 \text { August } 2019\end{array}$ \\
\hline Order: Atheriniforn & & \\
\hline Famil & & \\
\hline Xenentodon cancila(Hamilton, 1822) & Kankla & $\begin{array}{l}\text { Least Concern }(\mathrm{LC}) \text {; } \\
\text { assessed: } 12 \text { August } 2019\end{array}$ \\
\hline Order: Atheriniformes & & \\
\hline Family: Cyprinodontidae & & \\
\hline Aplocheilus panchax (Hamilton, 1822) & Tinchokh & $\begin{array}{l}\text { Least Concern (LC) ; } \\
\text { assessed: } 21 \text { June } 2018\end{array}$ \\
\hline Order: Channiformes & & \\
\hline Family: Channidae & & \\
\hline
\end{tabular}


Int J Adv Life Sci Res. Volume 4(2)14-22

\begin{tabular}{|c|c|c|}
\hline Channa marulius (Hamilton ,1822) & Shal & $\begin{array}{ccc}\text { Least } & \text { Concern } & (\mathrm{LC}) \\
\text { assessed: } & 06 \text { October } 2009\end{array}$ \\
\hline Channa punctata (Bloch, 1793) & Sati & $\begin{array}{l}\text { Least Concern }(\mathrm{LC}) \text {; } \\
\text { assessed:11 August } 2019\end{array}$ \\
\hline Channa striata (Bloch, 1793) & Shol & $\begin{array}{l}\text { Least Concern }(\mathrm{LC}) \\
\text { assessed:11 August } 2019\end{array}$ \\
\hline \multicolumn{3}{|l|}{ Order: Synbranchiformes } \\
\hline \multicolumn{3}{|l|}{ Family: Synbranchidae } \\
\hline Monopterus cuchia (Hamilton, 1822) & Cuche & $\begin{array}{l}\text { Least } \quad \text { Concern } \quad(\mathrm{LC}) \\
\text { assessed: } 20 \text { March } 2010\end{array}$ \\
\hline \multicolumn{3}{|l|}{ Order: Perciformes } \\
\hline \multicolumn{3}{|l|}{ Family: Chandidae } \\
\hline Chanda nama (Hamilton, 1822) & Chada & $\begin{array}{l}\text { Least } \quad \text { Concern }(\mathrm{LC}) \text {; } \\
\text { assessed: } 16 \text { March } 2010\end{array}$ \\
\hline Parambassis ranga (Hamilton, 1822) & Chada & $\begin{array}{l}\text { Least } \quad \text { Concern } \quad(\mathrm{LC}) \text {; } \\
\text { assessed: } 16 \text { March } 2011\end{array}$ \\
\hline Parambassis baculis (Hamilton, 1822) & Chada & $\begin{array}{l}\text { Least } \quad \text { Concern } \quad(\mathrm{LC}) ; \\
\text { assessed: } 20 \text { March } 2010\end{array}$ \\
\hline \multicolumn{3}{|l|}{ Family: Nandidae } \\
\hline Badis badis (Hamilton, 1822) & Bot koi & $\begin{array}{l}\text { Least } \quad \text { Concern } \quad(\mathrm{LC}) ; \\
\text { assessed: } 26 \text { March } 2010\end{array}$ \\
\hline Nandus nandus(Hamilton, 1822) & Nandos & $\begin{array}{l}\text { Least Concern } \quad(\mathrm{LC}) ; \\
\text { assessed: } 12 \text { October } 2009\end{array}$ \\
\hline \multicolumn{3}{|l|}{ Family: Cichlidae } \\
\hline Oreochromis niloticus (Linnaeus, 1758) & Nilontica & $\begin{array}{l}\text { Least } \quad \text { Concern } \quad(\mathrm{LC}) ; \quad \text { Date } \\
\text { assessed: } 02 \text { March 2018(Exotic) }\end{array}$ \\
\hline \multicolumn{3}{|l|}{ Family: Mugilidae } \\
\hline Rhinomugil corsula (Hamilton, 1822) & Khorsol & $\begin{array}{l}\text { Least Concern }(\mathrm{LC}) ; \\
\text { assessed: }\end{array}$ \\
\hline \multicolumn{3}{|l|}{ Family: Gobiidae } \\
\hline Glossogobius giuris (Hamilton, 1822) & Bele & $\begin{array}{l}\text { Least Concern (LC) } \\
\text { assessed: } 11 \text { August } 2019\end{array}$ \\
\hline \multicolumn{3}{|l|}{ Family: Anabantidae } \\
\hline Anabas testudineus (Bloch, 1792) & Koi & $\begin{array}{l}\text { Least } \quad \text { Concern } \quad(\mathrm{LC}) \\
\text { assessed: } 10 \text { August } 2019\end{array}$ \\
\hline \multicolumn{3}{|l|}{ Family: Belontidae } \\
\hline $\begin{array}{l}\text { Trichogaster fasciata } \\
\text { Schneider, 1801) }\end{array}$ & Kholse & $\begin{array}{l}\text { Least Concern } \quad(\mathrm{LC}) ; \\
\text { assessed: } 21 \text { January } 2010\end{array}$ \\
\hline Trichogaster lalius (Hamilton,1822) & Kholse & $\begin{array}{l}\text { Least Concern }(\mathrm{LC}) ; \\
\text { assessed: } 21 \text { January } 2010\end{array}$ \\
\hline Trichogaster chuna (Hamilton,1822) & Kholse & $\begin{array}{lcc}\text { Least } & \text { Concern } & (\mathrm{LC}) \\
\text { assessed: } & 12 \text { October } 2009\end{array}$ \\
\hline Trichogaster labiosa (Day, 1877) & Kholse & $\begin{array}{l}\text { Least Concern }(\mathrm{LC}) ; \\
\text { assessed: } 21 \text { January } 2010\end{array}$ \\
\hline \multicolumn{3}{|l|}{ Order: Tetraodontiformes } \\
\hline \multicolumn{3}{|l|}{ Family: Tetraodontidae } \\
\hline Leiodon cutcutia (Hamilton, 1822) & Tyapa & 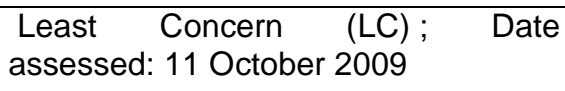 \\
\hline
\end{tabular}


Pie Chart showing Number of Species in Each Family

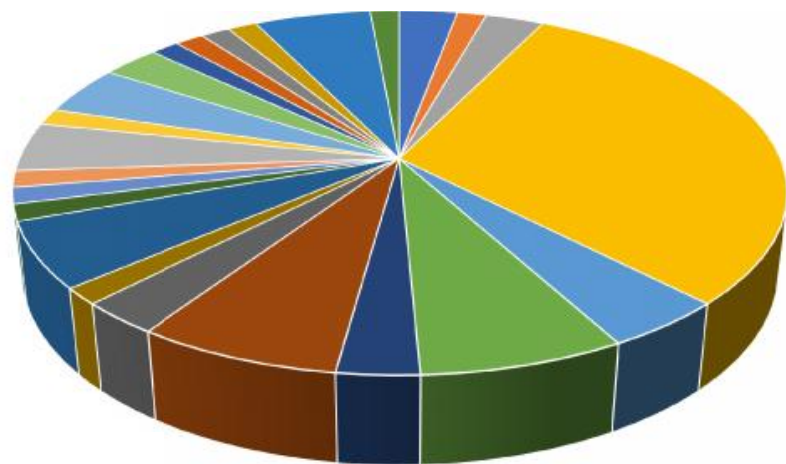

\begin{tabular}{|llll|}
\hline - Clupeidae & "Engraulidae & " Notopteridae & " Cyprinidae \\
- Cobitidae & " Bagridae & - Siluridae & - Schilbeidae \\
- Pangasiidae & - Amblycipitidae & - Sisoridae & - Heteropneustidae \\
- Belonidae & - Cyprinodontidae & - Channidae & "Synbranchidae \\
- Chandidae & - Nandidae & - Cichlidae & - Mugilidae \\
- Gobiidae & " Anabantidae & - Belontidae & - Tetraodontidae \\
\hline
\end{tabular}

Chart Showing Name of Orders with Number of Families and Species under each Order

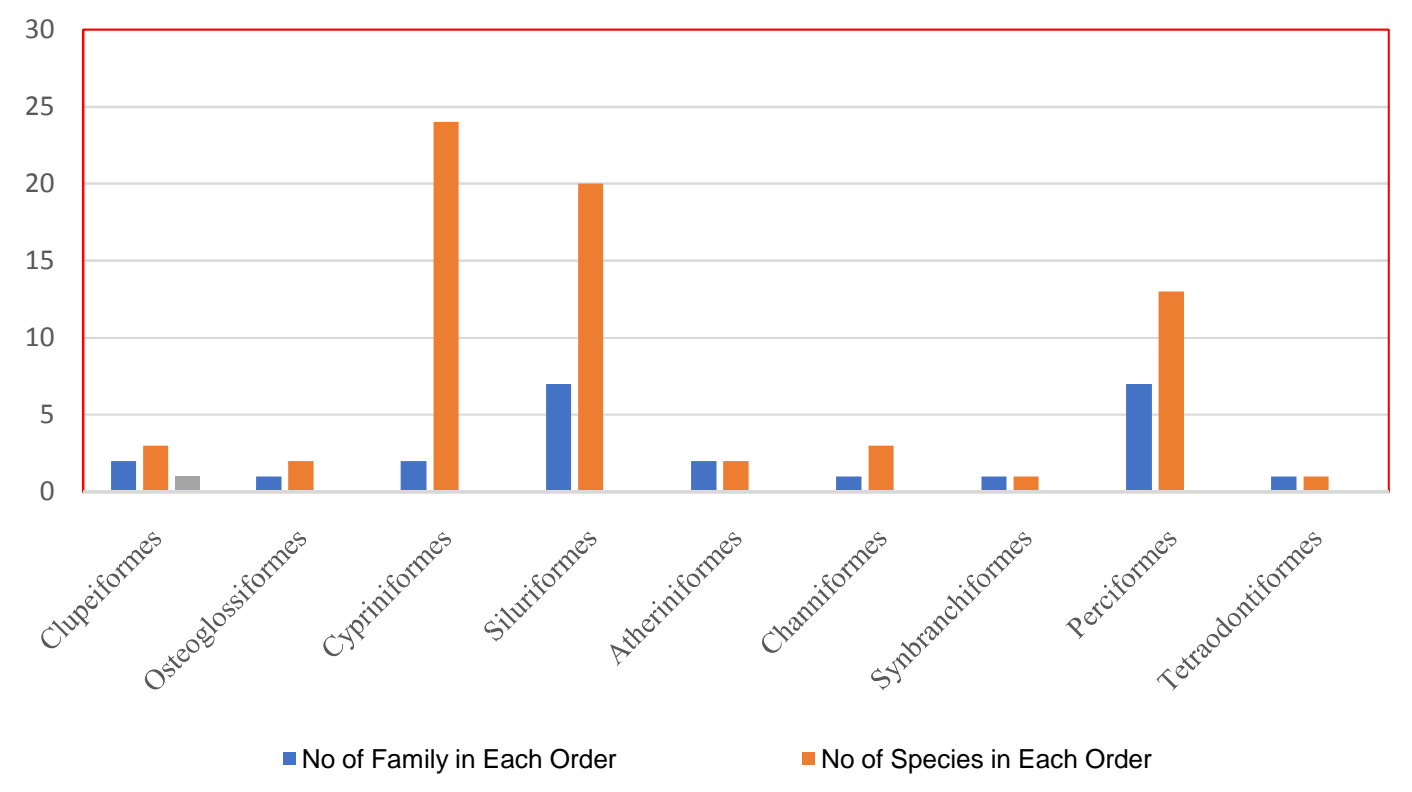


Table 2: Representing Fish Families with Number of Species Belonging to Them:

\begin{tabular}{|c|l|c|}
\hline $\begin{array}{c}\text { SI. } \\
\text { No. }\end{array}$ & Name of Family & $\begin{array}{c}\text { No. of } \\
\text { Species in } \\
\text { each Family }\end{array}$ \\
\hline 1 & Clupeidae & 2 \\
\hline 2 & Engraulidae & 1 \\
\hline 3 & Notopteridae & 2 \\
\hline 4 & Cyprinidae & 21 \\
\hline 5 & Cobitidae & 3 \\
\hline 6 & Bagridae & 5 \\
\hline 7 & Siluridae & 2 \\
\hline 8 & Schilbeidae & 5 \\
\hline 9 & Pangasiidae & 2 \\
\hline 10 & Amblycipitidae & 1 \\
\hline 11 & Sisoridae & 4 \\
\hline 12 & Heteropneustidae & 1 \\
\hline 13 & Belonidae & 1 \\
\hline 14 & Cyprinodontidae & 1 \\
\hline 15 & Channidae & 3 \\
\hline 16 & Synbranchidae & 1 \\
\hline 17 & Chandidae & 3 \\
\hline 18 & Nandidae & 2 \\
\hline 19 & Cichlidae & 1 \\
\hline 20 & Mugilidae & 1 \\
\hline 21 & Gobiidae & 1 \\
\hline 22 & Anabantidae & 1 \\
\hline 23 & Belontidae & 4 \\
\hline 24 & Tetraodontidae & 1 \\
\hline & & \\
\hline
\end{tabular}

Table 3: Representing Name of the Orders with Number of Families and Species found under each Order:

\begin{tabular}{|l|l|c|c|}
\hline $\begin{array}{l}\text { SI. } \\
\text { No. }\end{array}$ & Name of the Order & $\begin{array}{c}\text { No of } \\
\text { Family } \\
\text { in Each } \\
\text { Order }\end{array}$ & $\begin{array}{c}\text { No of } \\
\text { Speci } \\
\text { es in } \\
\text { Each } \\
\text { Order }\end{array}$ \\
\hline 1 & Clupeiformes & 2 & 3 \\
\hline 2 & Osteoglossiformes & 1 & 2 \\
\hline 3 & Cypriniformes & 2 & 24 \\
\hline 4 & Siluriformes & 7 & 20 \\
\hline 5 & Atheriniformes & 2 & 2 \\
\hline 6 & Channiformes & 1 & 3 \\
\hline 7 & Synbranchiformes & 1 & 1 \\
\hline 8 & Perciformes & 7 & 13 \\
\hline 9 & Tetraodontiformes & 1 & 1 \\
\hline
\end{tabular}

\section{Image of some Selected Fish}

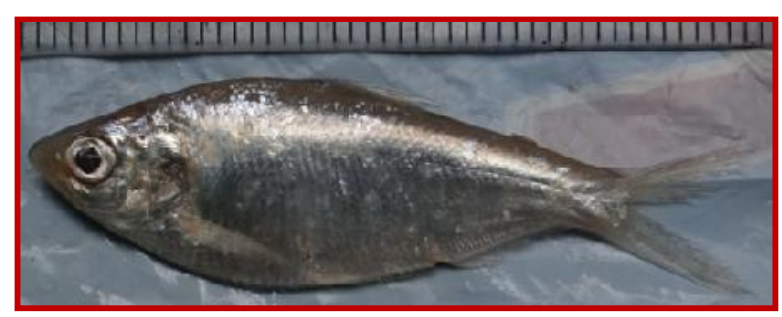

Gonialosa manmina

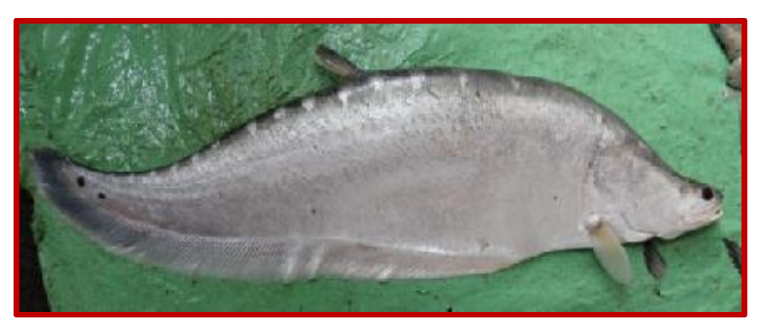

Chitala chitala

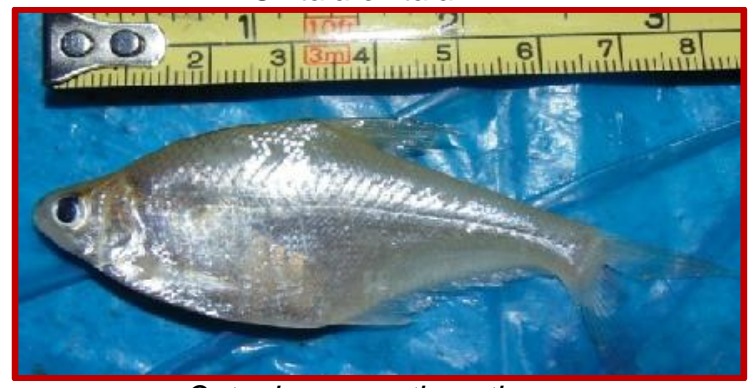

Osteobrama cotio cotio

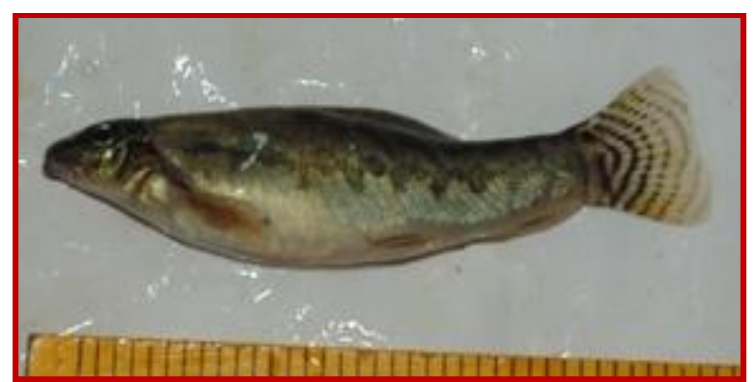

Acanthocobitis botia

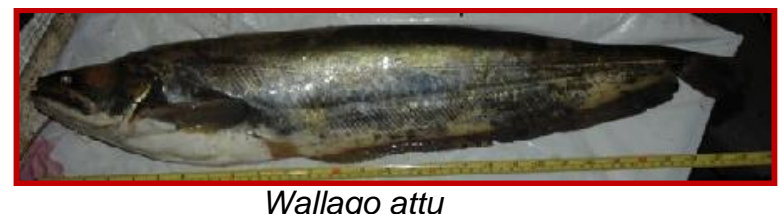

Wallago attu

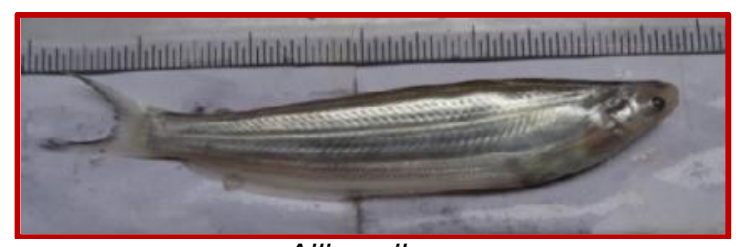

Ailia coila 


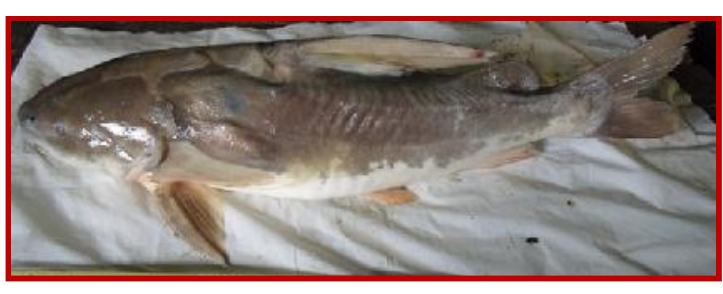

Rita rita

Discussion

The result showed that 69 freshwater fish species belonging to 9 Orders, 24 Families is found in Ganga stretch of Malda District. Out of 24 families Cyprinidae was found to be dominant having 21 species followed by Bagridae having 5 species. Order wise Cypriniformes contain 24 species followed by Siluriformes with 20 species and Perciformes with 13 species. Order Siluriformes contained 7 families and exhibited maximum number of families. In this study 3 fishes, Glyptothorax telchitta(Hamilton, 1822), Amblyceps apangi (Nath and Dey, 1989) and Garra annandalei (Hora, 1921) were found to be locally very rare though they all are in Least Concern (LC) category in IUCN list. 4 exotic species,

\section{References}

Barman, R.P. (2007), A review of the fresh water fish fauna of West Bengal, India with suggestions for conservation of the threatened and endemic species, Records of the Zoological Survey of India, Occasional Paper 263: 1-48.

Bilgrami K S, Datta Munshi J S and Verma S K (1992) Bioconservation and biomonitoring of river Ganga in Bihar. Final Technical Report, Ganga project Directorate, New Delhi.

Day, F. (1876). The Fishes of India: Being a Natural History of the Fishes known to inhabit the Seas and Fresh water of India, Burma and Ceylon. William Dawson \& Sons Ltd., London.778p.

Dwivedi A C, Mayank P and Tiwari A (2016) The River as transformed by human activities: the rise of the invader potential of Cyprinus carpio and Oreochromis niloticus from the Yamuna River, India. J. Earth Sci. Climatic Change 7,361. doi: 10.4172/ 2157-7617.1000361.

Jaya, Bhatta B., Baluni D.C. (2020), Study of Ichthyofaunal Diversity of Banghara Pokher Ghataho,Samastipur, Bihar. IJRASET. 8(XI), 393396.
Oreochromis niloticus (Linnaeus, 1758), Ctenopharyngodon idella (Valenciennes, 1844), Cyprinus carpio(Linnaeus, 1758) and Hypophthalmichthys molitrix (Valenciennes, 1844) were also found in this stretch of Ganga.

\section{Conclusion}

The objective of present study was to explore the present Ichthyofaunal diversity of the stretch of Ganga at Malda district of West Bengal. The result showed that 69 freshwater fish species belonging to 9 Orders, 24 Families was found in Ganga stretch of Malda District of West Bengal, India.

\section{Acknowledgments}

The authors are thankful to Principal, Rammohan College for her support in this study. This work was funded by West Bengal Bio-diversity Board, Kolkata, West Bengal, India.

\section{Conflicts of Interest}

The authors declare that there are no conflicts of interest.

Jayaram, K. C. (1981). The Freshwater Fishes of India: A Hand book. Zoological Survey of India, Calcutta. 475p.

Kumar.M, Choudhary S K, Varma M C(2019), Fish Fauna Distribution Pattern, Threats and Their Conservation Issues in Protected Areas: A Case Study from Vikramshila Gangetic Dolphin Sanctuary In Lower Ganga, Bihar, India. International Journal of Scientific \& Technology Research 8(9)12101217.

Mishra R. (1968). Ecology Work Book. New Delhi: Oxford and IBH Publishing Co., p. 197.

Nishok G., Guha T. L., \& Tatpati M. (2018). Community Based Conservation Amidst Conflict In The Dooars Region of North Bengal. www.kalpavriksh.org.

Pathak R K, Gopesh A, Dwivedi A C and Joshi K D (2014) Age and growth of alien fish species, Cyprinus carpio var. communis (Common carp) in the lower stretch of the Yamuna river at Allahabad. Natl Acad. Sci. Lett. 37, 419-422. DOI: 10.1007/ s40009-014-0262-3.

Pearce J. L., Schuurman D., Barber K. N., Larrivée M., Venier L. A., McKee J., \& McKenney D. (2005). 
Pitfall trap designs to maximize invertebrate captures and minimize captures of nontarget vertebrates. Canadian Entomologist, 137(2), 233250. https://doi.org/10.4039/n04-029.

Raunkiaer C. (1934). The Life Forms of Plants and Statistical Plant Geography. U. K.: Oxford University Press, p. 632.

Rodgers W.A., Panwar H.S. (1988). Planning a Wildlife Protected Area Network in India, 339, FAO, Dehra Dun , p. 267. 2vol. Project FO: IND/82/003.

Sagwal S.S. (1995). Forest Ecology of India. Jaipur: Pointer Publishers, pp. 87-111.

Sarkar U. K. Pathak A. K. Sinha R. K. , Sivakumar K. , Pandian A. K. , Pandey A. . Dubey V. K ,Lakra , W. S. ( 2012). Freshwater fish biodiversity in the River Ganga (India): Changing pattern, threats and conservation perspectives. Rev Fish Biol Fisheries . 22:251-272

Shannon C.E., Weaver (1949). The Mathematical Theory of Communication. Urbana, USA: University of Illinois Press, p. 117.
Shannon C.E., Weiner W. (1963). The Mathematical Theory of Communication. Urbana, Illinois, USA: University of Illinois Press, p. 111.

Singh, R.J. and Johal, M.S. (2009), Present status Fish diversity of the river Ganges in the vicinity of Allahabad, Uttar Pradesh, India. Acta Universitatis Carolinae Environmentalica 1-2(2009): 69-78

Singha Roy, U., Banerjee, P., \& Mukhopadhyay, S. K. (2012). Study on avifaunal diversity from three different regions of North Bengal, India. Asian Journal of Conservation Biology, 1(2), 120-129.

Sutherland W.J .2006. Ecological Census Techniques a handbook. Cambridge University Press, New York, 432pp.

Talwar, P. K. and Jhingran, A. G. (1991). Inland Fishes of India and Adjacent Countries (Vol. 1 \& 2). Oxford and IBH Publishing Co. Pvt. Ltd., Calcutta. $1158 p$ 